\title{
Effect of boundary vorticity discretization on explicit stream-function vorticity calculations
}

\author{
E. Sousa ${ }^{1, *, \dagger}$ and I. J. Sobey ${ }^{2}$ \\ ${ }^{1}$ Departamento de Matemática da Universidade de Coimbra, Portugal \\ ${ }^{2}$ Oxford University Computing Laboratory, U.K.
}

\begin{abstract}
SUMMARY
The numerical solution of the time-dependent Navier-Stokes equations in terms of the vorticity and a stream function is a well tested process to describe two-dimensional incompressible flows, both for fluid mixing applications and for studies in theoretical fluid mechanics. In this paper, we consider the interaction between the unsteady advection-diffusion equation for the vorticity, the Poisson equation linking vorticity and stream function and the approximation of the boundary vorticity, examining from a practical viewpoint, global iteration stability and error. Our results show that most schemes have very similar global stability constraints although there may be small stability gains from the choice of method to determine boundary vorticity. Concerning accuracy, for one model problem we observe that there were cases where the boundary vorticity discretization did not propagate to the interior, but for the usual cavity flow all the schemes tested had error close to second order. Copyright (c) 2005 John Wiley \& Sons, Ltd.
\end{abstract}

KEY WORDS: Navier-Stokes; vorticity boundary conditions; finite differences; stability

\section{INTRODUCTION}

While the majority of current fluid flow calculations are oriented towards three-dimensional flow fields, there remain an important group of simulations of two-dimensional unsteady laminar flows: either for applications which are approximately two dimensional or for the intrinsic relevance of two-dimensional flow to theoretical fluid mechanics, see for instance Reference [1]. In calculating two-dimensional flow there are significant advantages in using a streamfunction vorticity formulation of the Navier-Stokes equations rather than a velocity-pressure formulation: the continuity equation is automatically satisfied, only one advection equation has to be solved and there is no difficulty in matching a pressure to the velocity field. In

\footnotetext{
*Correspondence to: E. Sousa, Departamento de Matemática, Universidade de Coimbra, 3000-Coimbra, Portugal.

†E-mail: ecs@mat.uc.pt

Contract/grant sponsor: Centro de Matemática da Universidade de Coimbra
}

Copyright (c) 2005 John Wiley \& Sons, Ltd.

Received 17 June 2004

Revised 24 January 2005

Accepted 31 January 2005 
regular geometries (or irregular geometries using a conformal map) the use of a finite difference scheme to integrate the stream-function vorticity equations is straight forward except for one important area: the treatment of the boundary vorticity.

The purpose of this paper is to extend studies of the effect of different discretization of the vorticity boundary conditions for the unsteady incompressible Navier-Stokes equations in the stream-function vorticity formulation. We are especially interested in understanding the global stability properties of explicit time marching schemes using various methods for dealing with the spatially implicit vorticity boundary condition, although in principle, our methods could be applied to implicit time marching schemes. With this purpose we build a matrix form for the system of equations which couples together the advection-diffusion equation for the vorticity, the Poisson equation for the stream function and the vorticity boundary condition to provide a global iteration matrix; we then study the properties of that matrix for two particular flows. While stability is the primary focus of this work we also note in passing some results on accuracy which are in accordance with existing results although with some new data.

There are many studies of the stream function vorticity formulation for the Navier-Stokes equations and a substantial literature on the subject, from the seminal work of Thom [2] through to texts on computational fluid mechanics, ranging from the early text Reference [3] to the more recent Reference [4]. The basic method is also reviewed in References [5,6].

The way vorticity is handled at a boundary is extremely important from a physical point of view as it reflects the mechanism of vorticity generation at a boundary. The difficulty with a vorticity formulation is the lack of natural boundary condition on the vorticity since a no-slip boundary condition does not have a simple counterpart in terms of the vorticity. In order to complete the discrete formulation is nevertheless necessary to impose a numerical boundary condition on the vorticity. Perhaps the most well-known numerical vorticity boundary condition is that given by Thom [2] which comes from a quadratic polynomial approximation of the stream function near a boundary. The approximation is constrained to satisfy the correct normal derivative and then applied at the first interior point from the boundary. This type of numerical boundary condition has been analysed by Hou and Wetton [7], associated with a central scheme for the vorticity equation, and shown to yield second-order accurate solutions. Furthermore, Weinan and Liu [8] analysed fourth-order schemes associated to Briley's vorticity boundary condition leading to fourth-order accurate solutions. Later Wang and Liu [9] studied the Wilkes-Pearson formula associated with the central differences and a fourth-order scheme with Briley's formula. Other work that has focused on the role of vorticity boundary conditions is in for example: References [10] or [11]. More recently, $\mathrm{Li}$ and Wang [12] have generalized vorticity boundary conditions to curved boundary domains. There is also significant work on steady solutions and particularly their accuracy in Reference [13].

The plan of this paper is to briefly recap the formulation of the global iteration matrix for the Navier-Stokes equations (the idea was first explored in Reference [14] for a one-dimensional analogue of the stream-function vorticity equations), to consider a number of well-known discretizations (of both the vorticity transport equation and the boundary vorticity) and then to consider two cavity type flows. One is an exact solution of the Navier-Stokes equations and the other is a cavity flow driven by one wall moving uniformly. In each case we examine the variation of the solution with numerical parameters and the stability of explicit finite difference schemes for these flows. 


\section{GLOBAL ITERATION MATRIX FORMULATION}

In this section, we derive a global iteration matrix for the discretized form of the advectiondiffusion equation, the Poisson equation between the stream function and the vorticity and the boundary vorticity method, also considering briefly some conditions for stability of the global iteration.

\subsection{Flow equations}

We consider incompressible viscous flow in a two-dimensional domain without inflow or outflow. The motion of the fluid is governed by the Navier-Stokes equations,

$$
\begin{aligned}
\frac{\partial \mathbf{u}}{\partial t}+(\mathbf{u} \cdot \nabla) \mathbf{u} & =-\nabla p+\frac{1}{R e} \nabla^{2} \mathbf{u} \\
\nabla \cdot \mathbf{u} & =0
\end{aligned}
$$

where $\mathbf{u}=\mathbf{u}(x, y, t)=(u(x, y, t), v(x, y, t))$ is a non-dimensional velocity field, $p=p(x, y, t)$ is a non-dimensional pressure, and $R e$ is a Reynolds number. In a bounded domain $\Omega$ enclosed by a boundary $\partial \Omega$, the impermeability of the boundaries and the no-slip condition implies that

$$
\mathbf{u}(x, y, t)=\mathbf{u}_{W}(x, y, t) \quad \text { for }(x, y) \in \partial \Omega, t>0
$$

where $\mathbf{u}_{W}$ denotes the boundary velocity.

In terms of the vorticity field $\omega=v_{x}-u_{y}$, the momentum equations provide a vorticity equation,

$$
\frac{\partial \omega}{\partial t}+u \frac{\partial \omega}{\partial x}+v \frac{\partial \omega}{\partial y}=\frac{1}{R e} \nabla^{2} \omega
$$

The fluid velocity, $\mathbf{u}=(u, v)$ is obtained from

$$
u=\frac{\partial \psi}{\partial y}, \quad v=-\frac{\partial \psi}{\partial x}
$$

where $\psi(x, y, t)$ is a stream function, which is connected to the vorticity $\omega$, by a Poisson equation

$$
\omega=-\nabla^{2} \psi
$$

where without inflow or outflow in the cavity flows we consider, the stream function is zero on the boundary, $\psi_{\mid \partial \Omega}=0$. The boundary conditions (2) translate into boundary conditions for the stream function

$$
\left(\frac{\partial \psi}{\partial y},-\frac{\partial \psi}{\partial x}\right)=\mathbf{u}_{W}(x, y, t), \quad(x, y) \in \partial \Omega
$$

There is no explicit boundary condition for the vorticity. 


\subsection{Matrix form of discretization}

The idea of the matrix formulation was initially introduced in Reference [14] for a onedimensional model problem which was similar to a stream-function vorticity problem and so included some of the features of that problem but in other respects was considerably simpler, having only two boundary points. In two dimensions we have a more complex problem for various reasons, one of them being the fact that we have a considerably larger number of points on the boundary.

As we have noted, the stream-function vorticity formulation has the advantage that it not only eliminates the pressure variable, but also automatically enforces incompressibility. Yet, a difficulty in the numerical simulation of (3)-(6) is deciding a suitable numerical boundary condition for the vorticity. When the vorticity advection-diffusion equation is updated in time, that does not provide values for the vorticity on the boundaries, only at mesh points in the interior of $\Omega$ so that an additional condition is needed to determine the vorticity on the boundary.

We will describe how the problem (3)-(6) is implemented in matrix form. We assume that we are in a cavity, with $\Omega=[0,1] \times[0,1]$ but these ideas generalise straight forwardly to arbitrary domains and to more complicated flows with inlet and outlet conditions.

We start by writing the discretized vorticity values in two vectors, $\mathbf{W}_{\mathrm{I}}$, containing points which lie in the interior and $\mathbf{W}_{\mathrm{B}}$ containing points on the boundary. The discrete values of the stream function are similarly contained in $\boldsymbol{\Psi}_{\mathrm{I}}$, for the interior and $\boldsymbol{\Psi}_{\mathrm{B}}$ for the boundary. A time update of the vorticity advection-diffusion equation provides an update for the interior vorticity values only. A fairly broad class of time marching discretization for the vorticity advection equation, (3), can be written in the form

$$
\mathbf{Q} \mathbf{W}_{\mathrm{I}}^{n+1}=\mathbf{A} \mathbf{W}_{\mathrm{I}}^{n}+\mathbf{B} \mathbf{W}_{\mathrm{B}}^{n}
$$

for suitable matrices $\mathbf{Q}, \mathbf{A}$ and $\mathbf{B}$. Note that this formulation hides some aspects of the Navier-Stokes equations since according to (4), the matrices $\mathbf{A}$ and $\mathbf{B}$ are both functions of the stream function, and thus implicitly of the vorticity. Equation (7), covers some implicit and all explicit time marching schemes. For these cavity problems, assume there is uniform discretization where the space step is the same in both directions, namely $h$, and that the mesh points are

$$
\{(j h, k h): j, k=0, \ldots, m\}
$$

Thus the vector $\mathbf{W}_{\mathrm{I}}$ holds $(m-1)^{2}$ values and the vector $\mathbf{W}_{\mathrm{B}}$ has $4 m$ values. The dimensions of the matrices $\mathbf{Q}$ and $\mathbf{A}$ are $(m-1)^{2} \times(m-1)^{2}$ and of the matrix $\mathbf{B}$ are $(m-1)^{2} \times 4 m$.

For a driven cavity flow, the natural ordering of the stream-function values implies an ordering of values $\psi_{j k}=\psi(j h, k h), j, k=0, \ldots, m$ with $h=1 / m$. Suppose we denote a vector of values $\Psi_{j k}$ which approximate $\psi_{j k}$ using this natural ordering

$$
\boldsymbol{\Psi}=\left[\Psi_{00}, \Psi_{10}, \Psi_{20}, \ldots, \Psi_{m m}\right]^{\mathrm{T}}
$$

then there will exist a permutation matrix $\mathbf{P}$ such that

$$
\boldsymbol{\Psi}=\mathbf{P}\left[\begin{array}{l}
\boldsymbol{\Psi}_{\mathrm{B}} \\
\boldsymbol{\Psi}_{\mathrm{I}}
\end{array}\right]
$$


where $\boldsymbol{\Psi}_{\mathrm{B}}$ are values on the boundary ( $j$ or $k$ equal to 0 or $m$, in this case $4 m$ values), $\boldsymbol{\Psi}_{\mathrm{I}}$ those values in the interior $\left(0<j, k<m\right.$, in this case $(m-1)^{2}$ values $)$ and if $\mathbf{P}$ is suitable partitioned as $\mathbf{P}=\left[\mathbf{P}_{1}, \mathbf{P}_{2}\right]$ then

$$
\boldsymbol{\Psi}=\mathbf{P}_{1} \boldsymbol{\Psi}_{\mathrm{B}}+\mathbf{P}_{2} \boldsymbol{\Psi}_{\mathrm{I}}
$$

where the matrix $\mathbf{P}_{1}$ is a $(m+1)^{2} \times 4 m$ matrix and $\mathbf{P}_{2}$ is a $(m+1)^{2} \times(m-1)^{2}$ matrix.

In a similar manner the vorticity approximation $W_{j k}$, can be written (using the same permutation matrices)

$$
\mathbf{W}=\left[W_{00}, W_{10}, W_{20}, \ldots, W_{m m}\right]^{\mathrm{T}}=\mathbf{P}_{1} \mathbf{W}_{\mathrm{B}}+\mathbf{P}_{2} \mathbf{W}_{\mathrm{I}}
$$

Next, the stream function vorticity Poisson equation can be discretized at the interior points by

$$
\mathbf{R} \Psi=-h^{2} \mathbf{W}_{\mathrm{I}}
$$

where $\mathbf{R}$ is a matrix of dimension $(m-1)^{2} \times(m+1)^{2}$ and is easy to write down in terms of the natural ordering of $\boldsymbol{\Psi}$, so that in Equation (12)

$$
\begin{gathered}
\frac{1}{h^{2}} \mathbf{L} \Psi_{\mathrm{I}}^{n+1}+\frac{1}{h^{2}} \mathbf{N} \boldsymbol{\Psi}_{\mathrm{B}}^{n+1}=-\mathbf{W}_{\mathrm{I}}^{n+1} \\
\mathbf{L}=\mathbf{R} \mathbf{P}_{2} \quad \text { and } \quad \mathbf{N}=\mathbf{R} \mathbf{P}_{1}
\end{gathered}
$$

and $\mathbf{L}$ is a $(m-1)^{2} \times(m-1)^{2}$ and $\mathbf{N}$ is an $(m-1)^{2} \times 4 m$ matrix.

If the vorticity equation is discretized at the interior points (again using the natural ordering of $\boldsymbol{\Psi}^{n}$ ),

$$
\mathbf{Q} \mathbf{W}_{\mathrm{I}}^{n+1}=\mathbf{G} \mathbf{W}^{n}=\mathbf{G}\left[\mathbf{P}_{1} \mathbf{W}_{\mathrm{B}}^{n}+\mathbf{P}_{2} \mathbf{W}_{\mathrm{I}}^{n}\right]
$$

where $\mathbf{G}$ is a matrix of dimensions $(m-1)^{2} \times(m+1)^{2}$. Then

$$
\mathbf{Q} \mathbf{W}_{\mathrm{I}}^{n+1}=\mathbf{G} \mathbf{P}_{1} \mathbf{W}_{\mathrm{B}}^{n}+\mathbf{G} \mathbf{P}_{2} \mathbf{W}_{\mathrm{I}}^{n}
$$

so that the matrix $\mathbf{A}$ and $\mathbf{B}$ in (7) are determined by

$$
\mathbf{A}=\mathbf{G P}_{2} \text { and } \mathbf{B}=\mathbf{G P}_{1}
$$

The boundary vorticity is more complicated, since the discretization there relates the wall vorticity to the updates stream function, but if we use a natural ordering to obtain on the boundary

$$
\mathbf{D}_{1} \mathbf{W}^{n+1}=\frac{1}{h^{2}} \mathbf{D}_{2} \Psi^{n+1}+\mathbf{v}^{n+1}
$$

where $\mathbf{D}_{1}$ and $\mathbf{D}_{2}$ are $4 m \times(m+1)^{2}$ matrices. The vector $\mathbf{v}^{n+1}$ might for instance arise in a driven cavity problem where the walls are moving. It follows that

$$
\mathbf{D}_{1}\left[\mathbf{P}_{1} \mathbf{W}_{\mathrm{B}}^{n+1}+\mathbf{P}_{2} \mathbf{W}_{\mathrm{I}}^{n+1}\right]=\frac{1}{h^{2}} \mathbf{D}_{2}\left[\mathbf{P}_{1} \boldsymbol{\Psi}_{\mathrm{B}}^{n+1}+\mathbf{P}_{2} \boldsymbol{\Psi}_{\mathrm{I}}^{n+1}\right]+\mathbf{v}^{n+1}
$$

and so

$$
\mathbf{D}_{1} \mathbf{P}_{1} \mathbf{W}_{\mathrm{B}}^{n+1}=\frac{1}{h^{2}} \mathbf{D}_{2} \mathbf{P}_{1} \boldsymbol{\Psi}_{\mathrm{B}}^{n+1}+\frac{1}{h^{2}} \mathbf{D}_{2} \mathbf{P}_{2} \boldsymbol{\Psi}_{\mathrm{I}}^{n+1}-\mathbf{D}_{1} \mathbf{P}_{2} \mathbf{W}_{\mathrm{I}}^{n+1}+\mathbf{v}^{n+1}
$$


This can be rewritten

$$
\mathbf{W}_{\mathrm{B}}^{n+1}=\frac{1}{h^{2}} \mathbf{M} \boldsymbol{\Psi}_{\mathrm{I}}^{n+1}+\mathbf{F} \mathbf{W}_{\mathrm{I}}^{n+1}+\frac{1}{h^{2}} \mathbf{J} \boldsymbol{\Psi}_{\mathrm{B}}^{n+1}+\left(\mathbf{D}_{1} \mathbf{P}_{1}\right)^{-1} \mathbf{v}^{n+1}
$$

where the matrices $\mathbf{M}, \mathbf{F}$ and $\mathbf{J}$ are of dimensions $4 m \times(m-1)^{2}$ and given by

$$
\mathbf{M}=\left(\mathbf{D}_{1} \mathbf{P}_{1}\right)^{-1} \mathbf{D}_{2} \mathbf{P}_{2}, \quad \mathbf{F}=-\left(\mathbf{D}_{1} \mathbf{P}_{1}\right)^{-1} \mathbf{D}_{1} \mathbf{P}_{2}, \quad \mathbf{J}=\left(\mathbf{D}_{1} \mathbf{P}_{1}\right)^{-1} \mathbf{D}_{2} \mathbf{P}_{1}
$$

This enables the stream function to be eliminated in the interior using (13)

$$
\mathbf{W}_{\mathrm{B}}^{n+1}=\left(\mathbf{F}-\mathbf{M L}^{-1}\right) \mathbf{W}_{\mathrm{I}}^{n+1}+\frac{1}{h^{2}}\left(\mathbf{J}-\mathbf{M L}^{-1} \mathbf{N}\right) \boldsymbol{\Psi}_{\mathrm{B}}^{n+1}+\left(\mathbf{D}_{1} \mathbf{P}_{1}\right)^{-1} \mathbf{v}^{n+1}
$$

and then the update of the vorticity in the interior can be replaced so that

$$
\mathbf{W}_{\mathrm{B}}^{n+1}=\left(\mathbf{F}-\mathbf{M} \mathbf{L}^{-1}\right) \mathbf{Q}^{-1}\left(\mathbf{A} \mathbf{W}_{\mathrm{I}}^{n}+\mathbf{B} \mathbf{W}_{\mathrm{B}}^{n}\right)+\frac{1}{h^{2}}\left(\mathbf{J}-\mathbf{M} \mathbf{L}^{-1} \mathbf{N}\right) \boldsymbol{\Psi}_{\mathrm{B}}^{n+1}+\left(\mathbf{D}_{1} \mathbf{P}_{1}\right)^{-1} \mathbf{v}^{n+1}
$$

This essentially completes the derivation of the iteration matrix for this version of the Navier-Stokes equations, since we now have

$$
\left[\begin{array}{c}
\mathbf{W}_{\mathrm{I}}^{n+1} \\
\mathbf{W}_{\mathrm{B}}^{n+1}
\end{array}\right]=\left[\begin{array}{cc}
\mathbf{Q}^{-1} \mathbf{A} & \mathbf{Q}^{-1} \mathbf{B} \\
\left(\mathbf{F}-\mathbf{M L}^{-1}\right) \mathbf{Q}^{-1} \mathbf{A} & \left(\mathbf{F}-\mathbf{M L}^{-1}\right) \mathbf{Q}^{-1} \mathbf{B}
\end{array}\right]\left[\begin{array}{c}
\mathbf{W}_{\mathrm{I}}^{n} \\
\mathbf{W}_{\mathrm{B}}^{n}
\end{array}\right]+\mathbf{S}^{n+1}
$$

or

$$
\mathbf{W}^{n+1}=\mathbf{K} \mathbf{W}^{n}+\mathbf{S}^{n+1}
$$

where

$$
\mathbf{S}^{n+1}=\frac{1}{h^{2}}\left[\begin{array}{c}
0 \\
\left(\mathbf{J}-\mathbf{M L}^{-1} \mathbf{N}\right) \Psi_{\mathrm{B}}^{n+1}
\end{array}\right]+\left(\mathbf{D}_{1} \mathbf{P}_{1}\right)^{-1} \mathbf{v}^{n+1}
$$

and $\mathbf{K}$ denotes the overall iteration matrix,

$$
\mathbf{K}=\left[\begin{array}{cc}
\mathbf{Q}^{-1} \mathbf{A} & \mathbf{Q}^{-1} \mathbf{B} \\
\left(\mathbf{F}-\mathbf{M L}^{-1}\right) \mathbf{Q}^{-1} \mathbf{A} & \left(\mathbf{F}-\mathbf{M L}^{-1}\right) \mathbf{Q}^{-1} \mathbf{B}
\end{array}\right]
$$

Let $\mathbf{X}=\mathbf{F}-\mathbf{M L}^{-1}, p=(m-1)^{2}$ and $q=4 m$. We have

$$
\mathbf{K}_{(p+q)^{2}}=\left[\begin{array}{cc}
\mathbf{Q}^{-1} \mathbf{A}_{p \times p} & \mathbf{Q}^{-1} \mathbf{B}_{p \times q} \\
\mathbf{X} \mathbf{Q}^{-1} \mathbf{A}_{q \times p} & \mathbf{X} \mathbf{Q}^{-1} \mathbf{B}_{q \times q}
\end{array}\right]
$$

and we can observe that

$$
\mathbf{K}=\left[\begin{array}{cc}
\mathbf{I}_{p \times p} & \mathbf{0}_{p \times q} \\
\mathbf{X}_{q \times p} & \mathbf{I}_{q \times q}
\end{array}\right]\left[\begin{array}{cc}
\mathbf{Q}_{p \times p}^{-1} & \mathbf{0}_{p \times q} \\
\mathbf{0}_{q \times p} & \mathbf{I}_{q \times q}
\end{array}\right]\left[\begin{array}{cc}
\mathbf{A}_{p \times p} & \mathbf{B}_{p \times q} \\
\mathbf{0}_{q \times p} & \mathbf{0}_{q \times q}
\end{array}\right]
$$


Note that if we have an explicit scheme then $\mathbf{Q}=\mathbf{I}$ so that

$$
\mathbf{K}=\left[\begin{array}{ll}
\mathbf{I} & \mathbf{0} \\
\mathbf{X} & \mathbf{I}
\end{array}\right]\left[\begin{array}{ll}
\mathbf{A} & \mathbf{B} \\
\mathbf{0} & \mathbf{0}
\end{array}\right]
$$

and $\mathbf{X}$ represents the influence of the vorticity boundary conditions and Poisson's equation and $\mathbf{A}$ and $\mathbf{B}$ the influence of the vorticity equation.

In this work, we consider two-level time-integration schemes and constant boundary conditions so that $\mathbf{S}^{n+1}=\mathbf{S}$, is constant and so,

$$
\mathbf{W}^{n+1}=\mathbf{K}_{\mathbf{n}} \mathbf{W}^{n}+\mathbf{S}
$$

where $\mathbf{S}$ contains boundary values of the stream function and of the velocity field and in the case where the flow field is evolving or time dependent since then the global iteration matrix varies from iteration to iteration and so is denoted $\mathbf{K}_{\mathbf{n}}$.

In Section 4, we consider a case where $\mathbf{K}_{\mathbf{n}}$ is constant, $\mathbf{K}_{\mathbf{n}}=\mathbf{K}$. Since the flow field is constant and known in advance,

$$
\mathbf{W}^{n+1}=\mathbf{K} \mathbf{W}^{n}+\mathbf{S}
$$

For such a steady flow, we can denote the difference between the steady solution, $\mathbf{W}$ and the current iteration value, $\mathbf{W}^{n}$ as an error, $\mathbf{e}^{n}=\mathbf{W}-\mathbf{W}^{n}$.

In that case the error $\mathbf{e}^{n}$ satisfies the equation

$$
\mathbf{e}^{n+1}=\mathbf{K} \mathbf{e}^{n}=\mathbf{K}^{n+1} \mathbf{e}^{0}
$$

When $\mathbf{K}$ is constant, we have the following stability condition, see Reference [15].

Stability condition: In order for $\mathbf{W}^{n}$ to remain bounded and the scheme, defined by the operator $\mathbf{K}^{n+1}$, to remain stable, the infinite set of operators $\mathbf{K}^{n}$ has to be uniformly bounded. That is, we should have, in a selected norm, for finite $T$

$$
\left\|\mathbf{K}^{n}\right\|<C \quad \text { for } 0<n \Delta t<T
$$

where $C$ is independent of $n, \Delta t, h$.

The norm of $\mathbf{K}^{n}$ is often very difficult to analyse, and instead a necessary condition but not always sufficient condition can be obtained from an analysis of the eigenvalues of $\mathbf{K}$.

The condition $\rho(\mathbf{K}) \leqslant 1$ is necessary for the scheme implemented by (30) to be stable, where $\rho(\mathbf{K})$ is the spectral radius of $\mathbf{K}$.

We have the following result.

\section{Proposition 1}

The value $\lambda \neq 0$ is an eigenvalue of the matrix $\mathbf{K}$, defined by (28), if and only if is an eigenvalue of the matrix $\mathbf{A}+\mathbf{B X}$.

\section{Proof}

If $\lambda \neq 0$ is an eigenvalue of the matrix $\mathbf{K}$ then exists $\mathbf{z}$ such that $\mathbf{K} \mathbf{z}=\lambda \mathbf{z}$. From this, we get $\mathbf{A} \mathbf{z}_{1}+\mathbf{B} \mathbf{z}_{2}=\lambda \mathbf{z}_{1}$ and $\mathbf{X} \mathbf{A} \mathbf{z}_{1}+\mathbf{X B z _ { 2 }}=\lambda \mathbf{z}_{2}$, where $\mathbf{z}=\left[\begin{array}{ll}\mathbf{z}_{1} & \mathbf{z}_{2}\end{array}\right]^{\mathrm{T}}$. We have $\mathbf{X} \mathbf{z}_{1}=\mathbf{z}_{2}$ and then $\mathbf{A} \mathbf{z}_{1}+\mathbf{B X} \mathbf{z}_{1}=\lambda \mathbf{z}_{1}$ and $\lambda$ is an eigenvalue of $\mathbf{A}+\mathbf{B X}$. 
Reciprocally if $\lambda$ is an eigenvalue of $\mathbf{A}+\mathbf{B X}$ then there is $\mathbf{z}_{1}$ such that $\mathbf{A} \mathbf{z}_{1}+\mathbf{B X} \mathbf{z}_{1}=\lambda \mathbf{z}_{1}$. Therefore, $\lambda$ is an eigenvalue of $\mathbf{K}$, since we have $\mathbf{K z}=\lambda \mathbf{z}$, where $\mathbf{z}=\left[\begin{array}{ll}\mathbf{z}_{1} & \mathbf{X} \mathbf{z}_{1}\end{array}\right]^{\mathrm{T}}$.

We cannot easily get explicit conditions between the eigenvalues of $\mathbf{A}$ and the eigenvalues of $\mathbf{A}+\mathbf{B X}$, since neither $\mathbf{A}$ or $\mathbf{B X}$ are special matrices, namely symmetric or other. However, the fact that the eigenvalues of $\mathbf{K}$ are the eigenvalues of $\mathbf{A}+\mathbf{B X}$ shows us more clearly that the different choices of the vorticity boundary conditions, implicitly represented by $\mathbf{X}$, can affect the spectral radius of $\mathbf{K}$.

Also if we want to compute numerically the spectrum of $\mathbf{K}$ for large dimensions is more efficient to use the matrix $\mathbf{A}+\mathbf{B X}$.

In later sections we are also going to use a fourth-order Poisson discretization. Briefly, we point out the changes that occurs in the matrix formulation when using this discretization.

Formula (13) come as

$$
\frac{1}{h^{2}} \mathbf{L} \boldsymbol{\Psi}_{\mathrm{I}}^{n+1}+\frac{1}{h^{2}} \mathbf{N} \boldsymbol{\Psi}_{\mathrm{B}}^{n+1}=-\mathbf{T}_{\mathbf{1}} \mathbf{W}_{\mathrm{I}}^{n+1}-\mathbf{T}_{\mathbf{2}} \mathbf{W}_{\mathrm{B}}^{n}
$$

and then

$$
\boldsymbol{\Psi}_{\mathrm{I}}^{n+1}=-\mathbf{L}^{-1} \mathbf{N} \Psi_{\mathrm{B}}^{n+1}-h^{2} \mathbf{L}^{-1} \mathbf{T}_{\mathbf{1}} \mathbf{W}_{\mathrm{I}}^{n+1}-h^{2} \mathbf{L}^{-1} \mathbf{T}_{\mathbf{2}} \mathbf{W}_{\mathrm{B}}^{n}
$$

Consequently, (23) is now given by

$$
\mathbf{W}_{\mathrm{B}}^{n+1}=\left(\mathbf{F}-\mathbf{M L}^{-1} \mathbf{T}_{\mathbf{1}}\right) \mathbf{W}_{\mathrm{I}}^{n+1}-\mathbf{M L}^{-1} \mathbf{T}_{\mathbf{2}} \mathbf{W}_{\mathrm{B}}^{n}+\frac{1}{h^{2}}\left(\mathbf{J}-\mathbf{M} \mathbf{L}^{-1} \mathbf{N}\right) \boldsymbol{\Psi}_{\mathrm{B}}^{n+1}+\left(\mathbf{D}_{1} \mathbf{P}_{1}\right)^{-1} \mathbf{v}^{n+1}
$$

and then

$$
\mathbf{W}_{\mathrm{B}}^{n+1}=\left[\left(\mathbf{F}-\mathbf{M L}^{-1} \mathbf{T}_{\mathbf{1}}\right) \mathbf{Q}^{-1} \mathbf{A} \quad\left(\mathbf{F}-\mathbf{M} \mathbf{L}^{-1} \mathbf{T}_{\mathbf{1}}\right) \mathbf{Q}^{-1} \mathbf{B}-\mathbf{M} \mathbf{L}^{-1} \mathbf{T}_{\mathbf{2}}\right]\left[\begin{array}{c}
\mathbf{W}_{\mathrm{I}}^{n} \\
\mathbf{W}_{\mathrm{B}}^{n}
\end{array}\right]+\mathbf{S}^{n+1}
$$

\section{FINITE DIFFERENCE DISCRETIZATIONS}

In this section, we set out two different schemes for the unsteady vorticity advection-diffusion equation; one a second-order Lax-Wendroff type scheme and one a third order, Quickest type scheme. These schemes, which are forms of well-known schemes of the same name, were introduced in Reference [16]. We consider two schemes for discretizing a Poisson equation, one the usual second-order central difference scheme and one a fourth-order scheme (see Reference [17]). We also set out a number of well-known schemes for discretizing the boundary vorticity, that of References [2,18-21]. We note in passing some consequences for stability from the discretization of the advection-diffusion equation.

\subsection{Discretization of the vorticity advection-diffusion equation}

We use the difference operators,

$$
\Delta_{x 0} W_{j, k}=\frac{1}{2}\left(W_{j+1, k}-W_{j-1, k}\right)
$$




$$
\begin{aligned}
\delta_{x}^{2} W_{j, k} & =W_{j+1, k}-2 W_{j, k}+W_{j-1, k} \\
\Delta_{x-} W_{j, k} & =W_{j, k}-W_{j-1, k}
\end{aligned}
$$

with operators $\Delta_{y 0} W_{j, k}, \delta_{y}^{2} W_{j, k}, \Delta_{y-} W_{j, k}$ defined analogously.

Also define local numerical parameters,

$$
v_{x}=\frac{u \Delta t}{h}, \quad v_{y}=\frac{v \Delta t}{h}, \quad \mu=\frac{1}{R e} \frac{\Delta t}{h^{2}}
$$

where $\Delta t$ is the time-step so that the iteration time is $t_{n}=n \Delta t$.

We discretize the vorticity advection-diffusion equation $(3)$ on the $(m-1) \times(m-1)$ interior points using two different numerical schemes.

An explicit second-order numerical Lax-Wendroff type scheme is given by

$$
\begin{aligned}
W_{j k}^{n+1}= & W_{j k}^{n}-v_{x} \Delta_{x 0} W_{j k}^{n}+\left(\frac{1}{2} v_{x}^{2}+\mu\right) \delta_{x}^{2} W_{j k}^{n}-v_{y} \Delta_{y 0} W_{j k}^{n} \\
& +\left(\frac{1}{2} v_{y}^{2}+\mu\right) \delta_{y}^{2} W_{j k}^{n}+v_{x} v_{y} \Delta_{x 0} \Delta_{y 0} W_{j k}^{n}, \quad j, k=1, \ldots, m-1
\end{aligned}
$$

This scheme uses a nine point stencil and can be used independently of the direction of the velocity field $(u, v)$.

An explicit third-order Quickest type scheme is given by

$$
\begin{aligned}
W_{j k}^{n+1}= & W_{j k}^{n}-v_{x} \Delta_{x 0} W_{j k}^{n}-v_{y} \Delta_{y 0} W_{j k}^{n}+\left(\frac{1}{2} v_{x}^{2}+\mu\right) \delta_{x}^{2} W_{j k}^{n} \\
& +\left(\frac{1}{2} v_{y}^{2}+\mu\right) \delta_{y}^{2} W_{j k}^{n}+v_{x} v_{y} \Delta_{x 0} \Delta_{y 0} W_{j k}^{n} \\
& +\frac{1}{6} v_{x}\left(1-v_{x}^{2}-6 \mu\right) \delta \Delta_{x} W_{j k}^{n}+\frac{1}{6} v_{y}\left(1-v_{y}^{2}-6 \mu\right) \delta \Delta_{y} W_{j k}^{n} \\
& -v_{y}\left(\mu+\frac{1}{2} v_{x}^{2}\right) \delta_{x}^{2} \Delta_{y 0} W_{j k}^{n}-v_{x}\left(\mu+\frac{1}{2} v_{y}^{2}\right) \delta_{y}^{2} \Delta_{x 0} W_{j k}^{n} \quad j, k=1, \ldots, m-1
\end{aligned}
$$

where the operators $\delta \Delta_{x}$ and $\delta \Delta_{y}$ change according to the direction of the velocity field as described in Table I. This scheme uses a 11 point stencil.

In the case of a non-linear velocity field, we treat these difference expansions as local approximations and use the velocity components $(u, v)$ involved in the variables $v_{x}$ and $v_{y}$ at the central mesh point, $\left(x_{j}, y_{k}\right)$. For additional information on the derivation of these two schemes see Reference [16].

Table I. Definition of the operators $\delta \Delta_{x}$ and $\delta \Delta_{y}$.

\begin{tabular}{lcccc}
\hline & $u, v \geqslant 0$ & $u \geqslant 0, v \leqslant 0$ & $u \leqslant 0, v \geqslant 0$ & $u, v \leqslant 0$ \\
\hline$\delta \Delta_{x}$ & $\delta_{x}^{2} \Delta_{x-}$ & $\delta_{x}^{2} \Delta_{x-}$ & $\delta_{x}^{2} \Delta_{x+}$ & $\delta_{x}^{2} \Delta_{x+}$ \\
$\delta \Delta_{y}$ & $\delta_{y}^{2} \Delta_{y-}$ & $\delta_{y}^{2} \Delta_{y+}$ & $\delta_{y}^{2} \Delta_{y-}$ & $\delta_{y}^{2} \Delta_{y+}$ \\
\hline
\end{tabular}


If we retain the locally constant approximation then the stability of the iteration is from a linear problem and we can use von Neumann stability analysis for the Cauchy problem involving the advection-diffusion vorticity equation. Although we apply the von Neumann analysis to the schemes above assuming the coefficients are linear, the conditions obtained are at least necessary stability conditions for the non-linear problem. For this reason it is worthwhile to take it into consideration.

A von Neumann analysis in two dimensions for linear problems is a straightforward generalization of the one-dimensional case. The discrete Fourier decomposition in two-dimensions consists of the decomposition of the function into a Fourier series as

$$
U_{j k}^{n}=\sum_{\xi_{x}, \xi_{y}} \kappa^{n} \mathrm{e}^{\mathrm{i} \xi_{x} j \Delta x} \mathrm{e}^{\mathrm{i} \xi_{y} k \Delta y}
$$

where the range $\xi_{x}, \xi_{y}$ is defined separately for each direction, as in the one-dimensional case. The amplification factor is given by $\kappa$. The products $\xi_{x} \Delta x$ and $\xi_{y} \Delta y$ are often represented as a phase angle, namely, $\theta_{x}=\xi_{x} \Delta x, \theta_{y}=\xi_{y} \Delta y$. To obtain a von Neumann stability condition we insert the singular component $\kappa^{n} \mathrm{e}^{\mathrm{i} j \theta_{x}} \mathrm{e}^{\mathrm{i} k \theta_{y}}$ into the discretized scheme. The amplification factor is said to satisfy the von Neumann condition if there is a constant $K$ such that

$$
\left|\kappa\left(\theta_{x}, \theta_{y}\right)\right| \leqslant 1+K \Delta t \quad \forall \theta_{x}, \theta_{y} \in[0,2 \pi]
$$

As in the one-dimensional case, in practice we use the stronger condition

$$
\left|\kappa\left(\theta_{x}, \theta_{y}\right)\right| \leqslant 1 \quad \forall \theta_{x}, \theta_{y} \in[0,2 \pi]
$$

and the discrete scheme that meets this condition, we refer to as von Neumann stable. This has been called practical stability by Richtmyer and Morton [15] or strict stability by other authors. In some cases condition (38) allows numerical modes to grow exponentially in time for finite values of $\Delta t$. Therefore, the practical, or strict, stability condition (39) is recommended in order to prevent numerical modes from growing faster than the physical modes of the differential equation.

For our finite difference schemes we have the following results, that are also represented in Figures 1 and 2.

\section{Proposition 2}

For the hyperbolic problem, that is, $\mu=0$, we have:

(a) Scheme (36) is stable if and only if

$$
\left|v_{x}\right|^{2 / 3}+\left|v_{y}\right|^{2 / 3} \leqslant 1
$$

(b) Scheme (37) is stable only if

$$
\left|v_{x}\right|+\left|v_{y}\right| \leqslant 1
$$

Proof

(a) This is well known, see Reference [22].

(b) Let $u, v \geqslant 0$ so that $v_{x}, v_{y} \geqslant 0$. The amplification factor for the phase angles $\theta_{x}=0$ and $\theta_{y}=\pi$ gives that to have $|\kappa| \leqslant 1$ is the same as to have $v_{x} \leqslant 1$. Similarly for the phase angles $\theta_{x}=\pi$ and $\theta_{y}=0$, we have $v_{y} \leqslant 1$.Then assuming $v_{x}, v_{y} \leqslant 1$ for the phase angles of high 

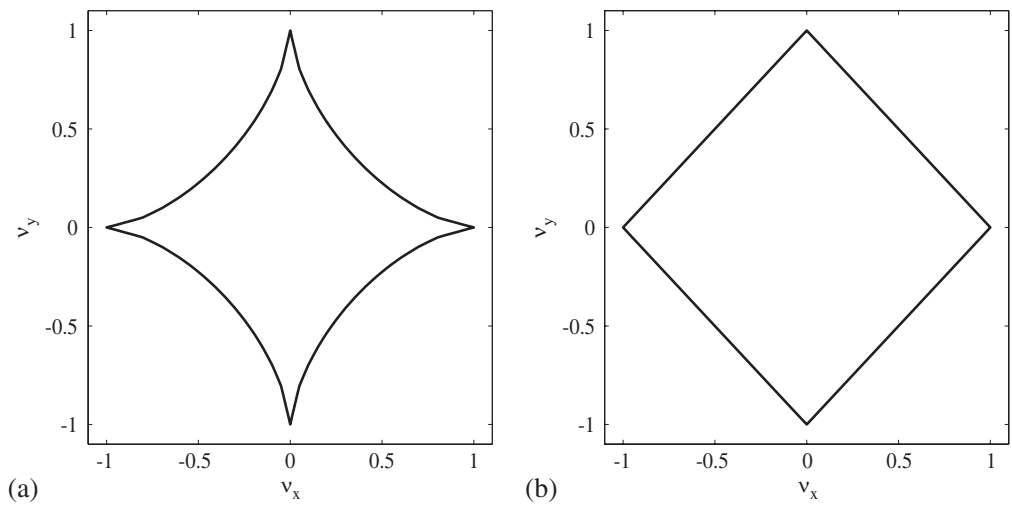

Figure 1. Stability conditions given in Proposition 2: (a) second-order scheme; and (b) third-order scheme.
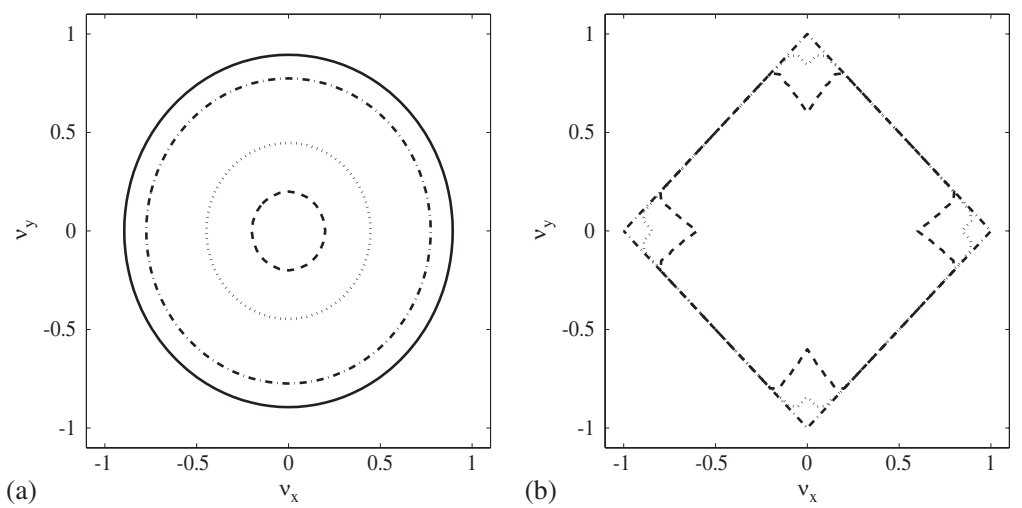

Figure 2. Stability conditions given in Proposition 3: (a) second-order scheme: contours for $\mu=0.05(-) ; \mu=0.1(-\cdot-) ; \mu=0.2(\cdots) ; \mu=0.24(--)$; and (b) third-order scheme: contours for $\mu=0.1(-\cdot-) ; \mu=0.2(\cdots) ; \mu=0.24(--)$.

frequency $\theta_{x}=\theta_{y}=\pi,|\kappa| \leqslant 1$ is equivalent to $v_{x}+v_{y} \leqslant 1$. Similarly results could be obtained for different directions of the velocity field in order to obtain condition (41).

\section{Proposition 3}

(a) Necessary von Neumann conditions for the stability of scheme (36) are

$$
4 \mu \leqslant 1, \quad v_{x}^{2}+v_{y}^{2} \leqslant 1-4 \mu
$$

(b) A necessary von Neumann condition for the stability of scheme (37) is

$$
\left(v_{x}^{2}+2 \mu\right)+\left(v_{y}^{2}+2 \mu\right)+\frac{2}{3}\left|v_{x}\right|\left(1-v_{x}^{2}-6 \mu\right)+\frac{2}{3}\left|v_{y}\right|\left(1-v_{y}^{2}-6 \mu\right) \leqslant 1
$$


Proof

(a) The first condition is obtained in the limiting case $\theta_{x} \rightarrow 0, \theta_{y} \rightarrow 0$. The second condition is obtained from the particular case $\theta_{x}=\theta_{y}=\pi$.

(b) Let $u, v \geqslant 0$ so that $v_{x}, v_{y} \geqslant 0$. The second condition is obtained from the particular case $\theta_{x}=\theta_{y}=\pi$.

\subsection{Discretization of the Poisson equation}

We consider two discretizations of the Poisson equation, (5). The first is the usual secondorder central difference scheme,

$$
\left(\delta_{x}^{2}+\delta_{y}^{2}\right) \Psi_{j, k}^{n+1}=-h^{2} W_{j, k}^{n+1}
$$

The second discretization was originated by Collatz [23], see also Reference [17],

$$
\left(\delta_{x}^{2}+\delta_{y}^{2}+\frac{1}{6} \delta_{x}^{2} \delta_{y}^{2}\right) \Psi_{j, k}^{n+1}=-h^{2}\left(I+\frac{1}{12}\left(\delta_{x}^{2}+\delta_{y}^{2}\right)\right) W_{j, k}^{n+1}
$$

where $I$ is an identity operator. This scheme is fourth-order accurate in $h$. We believe that this scheme is the same as that advocated in Reference [13] who derived the scheme afresh using discrete approximation for high-order derivatives in the truncation error.

\subsection{Boundary vorticity discretization}

While there are no formal or explicit conditions on the vorticity at a wall, it is necessary to use an implicit condition in order to provide the vorticity at the wall. There are various different methods for specifying wall vorticity, $\mathbf{W}_{\mathrm{B}}$, in terms of the vorticity in the interior and the stream function. An extensive review of methods for dealing with the boundary vorticity can be found in Reference [11]. In terms of our matrix formulation, different boundary vorticity schemes imply changes only in the matrices $\mathbf{D}_{1}, \mathbf{D}_{2}$.

The conditions we use to calculate the vorticity on the boundaries are illustrated for one boundary, $y=0$. Since the velocity field on that boundary, $u(x, 0)$, is not zero, the formulae assume that $\psi_{y}=u$ implicitly on the boundary. The formulae below are due to Thom [2], Woods [18], Jensen [19], d'Alessio and Dennis [20] and Briley [21].

$$
\begin{aligned}
\text { Thom: } W_{j 0} & =\frac{2}{h^{2}}\left(\Psi_{j 0}-\Psi_{j 1}+h u(j h, 0)\right) \\
\text { Woods: } W_{j 0} & =\frac{3}{h^{2}}\left(\Psi_{j 0}-\Psi_{j 1}+h u(j h, 0)\right)-\frac{1}{2} W_{j 1} \\
\text { Jensen: } W_{j 0} & =\frac{1}{2 h^{2}}\left(7 \Psi_{j 0}-8 \Psi_{j, 1}+\Psi_{j 2}+6 h u(j h, 0)\right) \\
\text { D'Alessio and Dennis: } W_{j 0} & =\frac{4}{h^{2}}\left(\Psi_{j 0}-\Psi_{j 1}+h u(j h, 0)\right)-\frac{1}{3}\left(4 W_{j 1}-W_{j 2}\right) \\
\text { Briley: } W_{j 0} & =\frac{1}{18 h^{2}}\left(85 \Psi_{j, 0}-108 \Psi_{j, 1}+27 \Psi_{j, 2}-4 \Psi_{j, 3}+66 h u(j h, 0)\right)
\end{aligned}
$$


We omit the superscript $n+1$ defining the time, $t_{n+1}$, in formulas (44)-(48), all the variables are taken at the updated time level, $t_{n+1}$. The discretization for the other boundaries is analogous. Considering (18) we have for Thom's vorticity boundary condition that $\mathbf{D}_{1}=\mathbf{I}$ where $\mathbf{I}$ is the matrix identity and $\mathbf{D}_{2}$ depends on the values of $\Psi$ at the boundary discretization. For Woods' and d'Allesio and Dennis' boundary condition $\mathbf{D}_{1}$ is no longer an identity matrix. Also for Thom's, Jensen's and Briley's formulae, $\mathbf{F}=\mathbf{0}$, since they do not depend on the interior vorticity values. The order of accuracy of the various methods (see Reference [11]) is $O(h)$ for Thom's, $O\left(h^{2}\right)$ for Woods', Jensen's and d'Alessio and Dennis' and $O\left(h^{3}\right)$ for Briley's.

\section{CAVITY FLOW WHICH IS EXACT SOLUTION OF NAVIER-STOKES EQUATIONS}

In this section, we approach the problem of using the global iteration matrix by turning to an exact solution of the Navier-Stokes equations. The solution is somewhat contrived in that it relies on a body force which may not be attainable in reality but it is, nevertheless, an exact solution. This allows consideration of precisely defined error measures, whereas normally in dealing with flow problems, one can only test against solutions obtained from refined meshes.

We start from the stream function

$$
\psi(x, y)=\frac{1}{\pi} \sin \pi x \sin \pi y
$$

which will take constant (zero) value on the boundaries of the unit square and so in one sense can be described as a driven cavity problem. This stream-function describes a flow with velocity field $\mathbf{u}=(u, v)$

$$
\begin{aligned}
& u(x, y)=\sin \pi x \cos \pi y \\
& v(x, y)=-\cos \pi x \sin \pi y
\end{aligned}
$$

and vorticity

$$
\omega=-\nabla^{2} \psi=2 \pi \sin \pi x \sin \pi y
$$

In order to make this an exact solution, consider the momentum equation in the Navier-Stokes equations, (1), with the addition of a body force, $\mathbf{f}$,

$$
\frac{\partial \mathbf{u}}{\partial t}+(\mathbf{u} \cdot \nabla) \mathbf{u}=-\nabla p+\frac{1}{R e} \nabla^{2} \mathbf{u}+\mathbf{f}
$$

and choose

$$
\begin{aligned}
& p=\frac{1}{4}(\cos 2 \pi x+\cos 2 \pi y) \\
& \mathbf{f}=\frac{2 \pi^{2}}{R e}[\sin \pi x \cos \pi y,-\cos \pi x \sin \pi y]
\end{aligned}
$$

The set $\psi, p$ and $\mathbf{f}$ provide an exact solution to the steady Navier-Stokes equations. 
The problem we consider is to suppose that we have some distribution of vorticity $W(x, y, t)$ and stream function, $\Psi(x, y, t)$ related by

$$
\nabla^{2} \Psi=-W
$$

but subject to the steady velocity field $\mathbf{u}$ and body force $\mathbf{f}$ through the vorticity advection equation which results from (53). The time dependent Navier-Stokes equations will give $W \rightarrow \omega$ as $t \rightarrow \infty$ and we let $W$ satisfy:

$$
\frac{\partial W}{\partial t}+(\mathbf{u} \cdot \nabla) W=\frac{1}{R e} \nabla^{2} W-\frac{1}{R e} \nabla^{2} \omega
$$

Now use the result that for this exact steady flow field

$$
\frac{\partial \omega}{\partial t} \equiv 0 \quad(\mathbf{u} \cdot \nabla) \omega \equiv 0
$$

so that the difference, $e=W-\omega$, satisfies

$$
\frac{\partial e}{\partial t}+(\mathbf{u} \cdot \nabla) e=\frac{1}{R e} \nabla^{2} e
$$

We interpret $e$ as an error in a vorticity field, the error satisfying an advection-diffusion equation with a velocity field which is spatially varying but constant in time. If the vorticity time variation on the boundary is set to zero, then the vorticity error will decay to zero in time as $W \rightarrow \omega$ and properties of the discretization of the advection-diffusion equation alone should determine the vorticity time variation behaviour in time. If, however, the boundary vorticity is not specified explicitly, but determined as usual for the stream-function vorticity formulation, then the error will not decay to zero in time but will reflect a global truncation error of the discretization of the whole stream-function vorticity system. Hence, the procedure to carry out one time step is: (a) update the error $e$ in the interior of the domain using (59), (b) calculate $W$ in the interior from $W=e+\omega$, (c) calculate the stream function, $\Psi$ in the interior by solving $\nabla^{2} \Psi=-W$, (d) calculate the vorticity $W$ on the boundary using an appropriate method, and finally (e) calculate the error on the boundary using $e=W-\omega$.

There are two numerical parameters which characterize the system,

$$
v=\frac{\Delta t}{h}, \quad \mu=\frac{1}{\operatorname{Re}} \frac{\Delta t}{h^{2}}
$$

\subsection{Stability}

We consider consequences for stability of choosing different boundary vorticity conditions. Since the vorticity difference, $e$, satisfies an advection-diffusion equation with constant velocity field we can examine stability through the global iteration matrix, $\mathbf{K}$, defined in (27), where we used the second-order Poisson's discretization.

The results for the eigenvalues of $\mathbf{K}$ for different vorticity boundary conditions with the second-order Lax-Wendroff type scheme are shown in Figure 3 where we also show the eigenvalues of the iteration matrix for the advection-diffusion equation, $\mathbf{A}$, The results were obtained for the mesh size $m=32$. The overall pattern is that there may be a small reduction in the region of stability with higher order methods but in general, the choice of boundary 

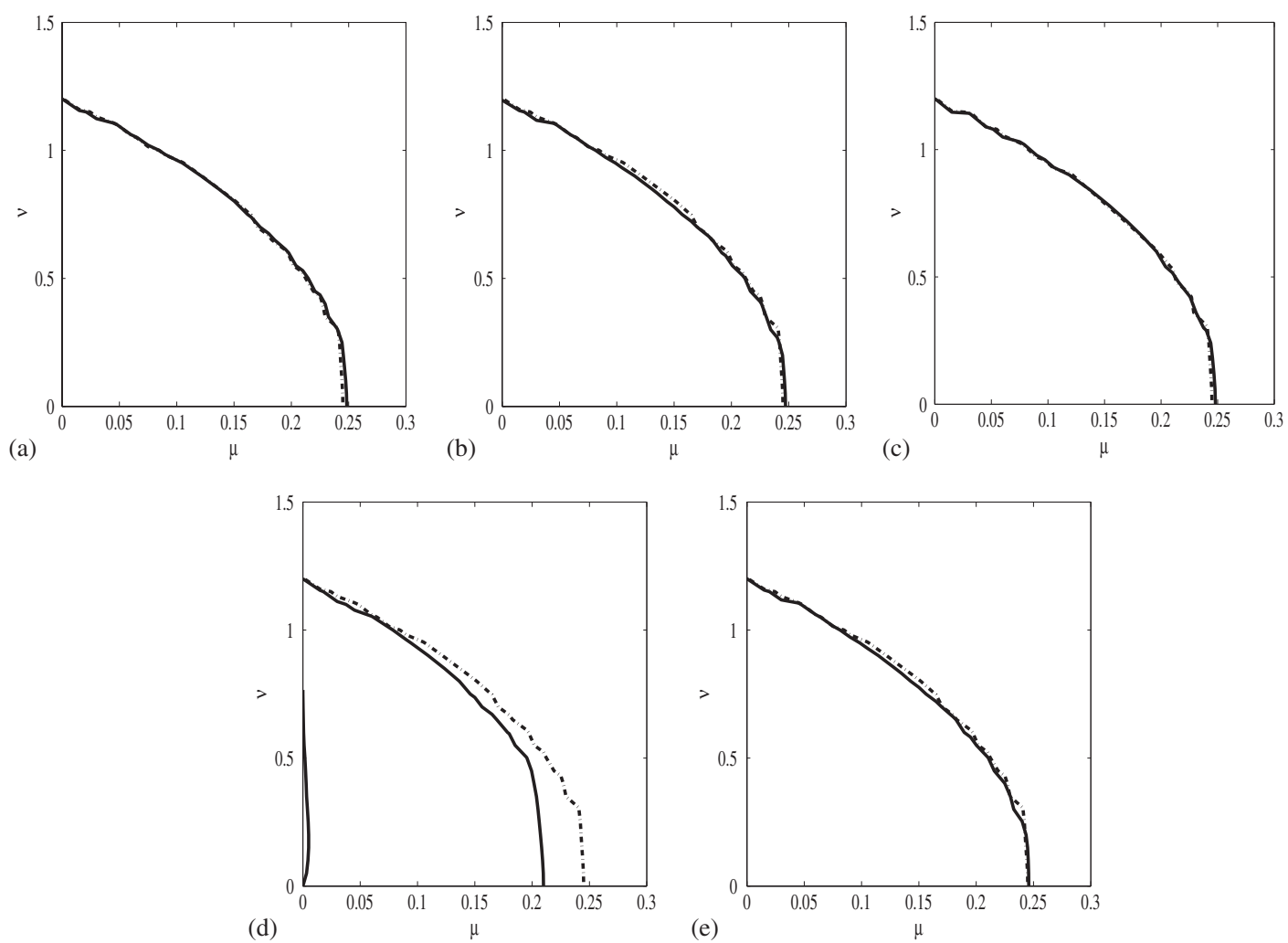

Figure 3. Eigenvalues less than one: the local matrix $A(\cdot-)$; the global matrix $K(-)$; $m=32$. Second-order method for advection-diffusion with: (a) Thom's boundary condition; (b) Woods's boundary condition; (c) Jensen boundary condition; (d) Alessio's boundary condition; and (e) Briley's boundary condition.

vorticity discretization does not have significant stability penalties. Mesh refinement does not affect this conclusion.

We have also considered the case of a third-order Quickest type scheme for the advectiondiffusion equation and the regions of stability are shown in Figure 4. In this case there is a more noticeable stability penalty but again, only marginally important. There is a region near the $\mu=0$ axis where the eigenvalues are predicted to be very slightly greater than one but in practical computations, the iterations remain stable in this region.

Although Figures 3 and 4 display the stability regions obtained when using the secondorder Poisson's discretization, for the fourth-order Poisson discretization (43) we obtain very similar stability regions.

\subsection{Accuracy}

We have used solution of system (56) and (59) to examine the accuracy of the different numerical schemes although as we shall see in the next section, the conclusions are more limited than we had hoped. 

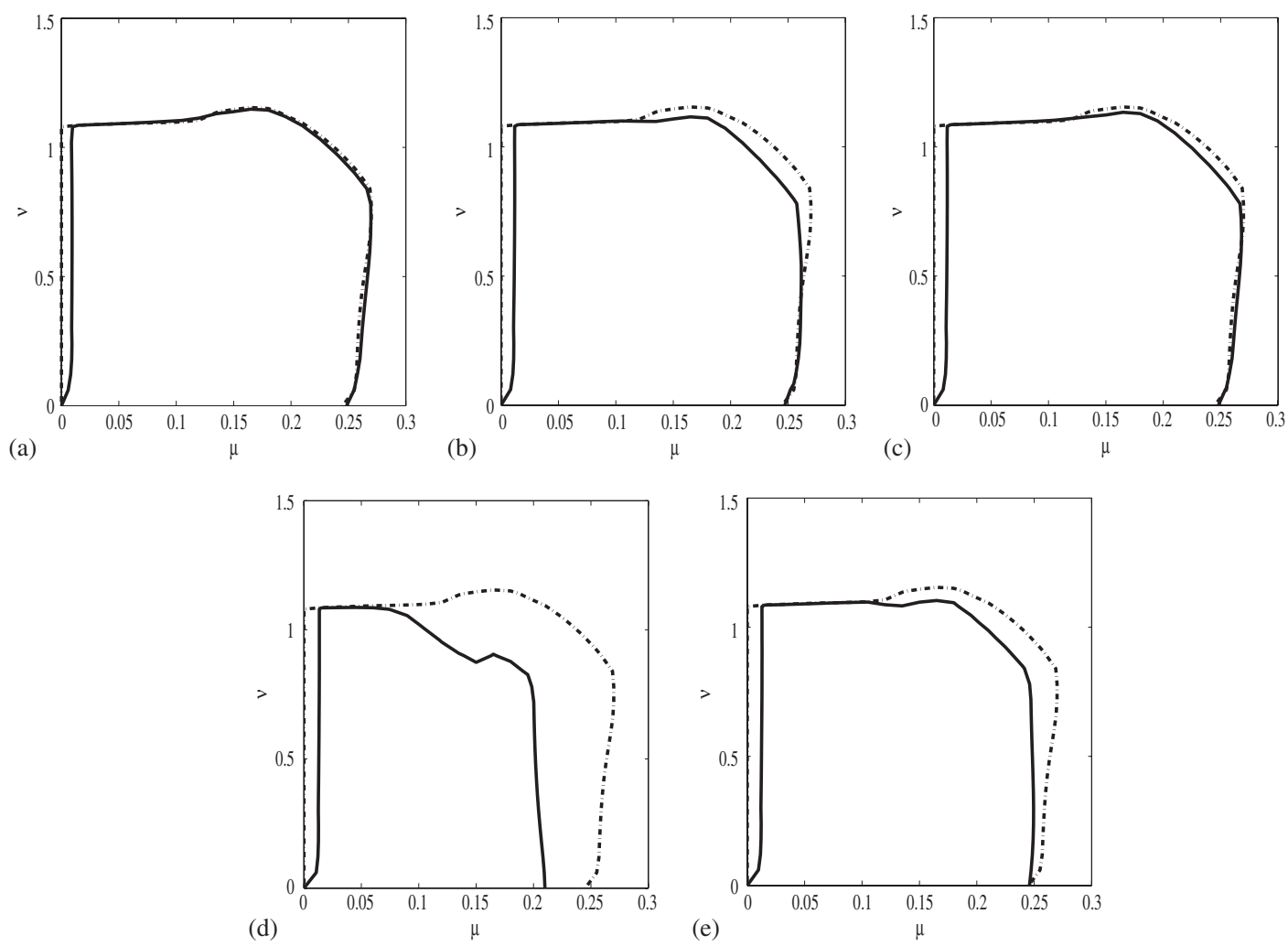

Figure 4. Eigenvalues less than one: the local matrix $A(\cdot-)$; the global matrix $K$ $(-) ; m=32$. Third-order method for advection-diffusion with: (a) Thom's boundary condition; (b) Wood's boundary condition; (c) Jensen boundary condition; (d) Alessio's boundary condition; and (e) Briley's boundary condition.

We start the iteration with the vorticity initially set to have unit value everywhere except at the four corner nodes where it is set to zero (the corner values of the vorticity are not used anywhere in the iteration). The system is then updated according to the scheme described above with the following possible choices:

(i) Lax-Wendroff or Quickest for advection-diffusion equation,

(ii) a second- or fourth-order scheme for solution of the Poisson equation,

(iii) boundary vorticity scheme from: Thom, Woods, Jensen, d'Allessio and Dennis or Briley.

The Poisson equation was solved using a four-level multigrid solver with convergence criterion set to $L_{\infty}$ norm of the residual less than $10^{-7}$. As the global error is converging to zero as the mesh size vanishes, the order of convergence can be extrapolated using two meshes. In the results we do this for $16 \times 16$ and $32 \times 32$ meshes and for $32 \times 32$ and $64 \times 64$ meshes.

The results shown in Table II are a little surprising since for the majority of cases secondorder convergence is obtained regardless of the discretization of the vorticity equation or which 
form of boundary condition is used, however, in the case of Jensen or Briley's method, fourthorder convergence is obtained when the Poisson solver is also fourth order. We believe that this is consistent with the theory of Bramble and Hubbard [24] who showed that for an elliptic problem with truncation error $O\left(h^{n}\right)$ [h is mesh spacing] then the global error would remain $O\left(h^{n}\right)$ when there were local errors of order $O\left(h^{n-1}\right)$ near a boundary with a mixed or Neumann condition and $O\left(h^{n-2}\right)$ near a boundary with Dirichlet condition. The complexity of this particular problem means that we cannot prove this result formally at present. It is also surprising that the discretization of the vorticity equation does not affect the numerical results but that too may be consistent with the theory of Bramble and Hubbard [24] or it may be a consequence of the very artificial nature of this test problem. Since in the next section where we consider a driven cavity flow, we do not see this behaviour, it is most likely that the convergence here is determined more by the solution of Poisson equation than by the solution of the advection-diffusion equation so that the correct explanation for the global convergence rate comes from Bramble-Hubbard theory.

Table II. Global $L_{2}$ error of time converged solution for three mesh resolutions, $16 \times 16,32 \times 32$ and $64 \times 64$, with calculated convergence rate for varying vorticity boundary condition, convection-diffusion discretization and discretization of stream function-vorticity equation.

\begin{tabular}{|c|c|c|c|c|c|c|c|}
\hline \multirow{2}{*}{$\begin{array}{l}\text { Boundary } \\
\text { method }\end{array}$} & \multirow[b]{2}{*}{ Convection-diffusion } & \multirow{2}{*}{$\begin{array}{l}\text { Poisson } \\
\text { equation }\end{array}$} & \multicolumn{3}{|c|}{ Error } & \multicolumn{2}{|c|}{ Convergence } \\
\hline & & & $16 \times 16$ & $32 \times 32$ & $64 \times 64$ & $16-32$ & $32-64$ \\
\hline Thom & $\begin{array}{c}\text { Lax-Wendroff } \\
\text { Quickest }\end{array}$ & $\begin{array}{l}O\left(h^{2}\right) \\
O\left(h^{4}\right) \\
O\left(h^{2}\right) \\
O\left(h^{4}\right)\end{array}$ & $\begin{array}{c}0.914 \mathrm{E}-02 \\
0.181 \mathrm{E}-01 \\
0.909 \mathrm{E}-02 \\
0.180 \mathrm{E}-01\end{array}$ & $\begin{array}{l}0.222 \mathrm{E}-02 \\
0.443 \mathrm{E}-02 \\
0.222 \mathrm{E}-02 \\
0.443 \mathrm{E}-02\end{array}$ & $\begin{array}{l}0.546 \mathrm{E}-03 \\
0.109 \mathrm{E}-02 \\
0.546 \mathrm{E}-03 \\
0.109 \mathrm{E}-02\end{array}$ & $\begin{array}{l}2.04 \\
2.03 \\
2.03 \\
2.02\end{array}$ & $\begin{array}{l}2.02 \\
2.02 \\
2.02 \\
2.02\end{array}$ \\
\hline Woods & $\begin{array}{c}\text { Lax-Wendroff } \\
\text { Quickest }\end{array}$ & $\begin{array}{l}O\left(h^{2}\right) \\
O\left(h^{4}\right) \\
O\left(h^{2}\right) \\
O\left(h^{4}\right)\end{array}$ & $\begin{array}{l}0.278 \mathrm{E}-01 \\
0.183 \mathrm{E}-01 \\
0.275 \mathrm{E}-01 \\
0.181 \mathrm{E}-01\end{array}$ & $\begin{array}{c}0.668 \mathrm{E}-02 \\
0.444 \mathrm{E}-02 \\
0.667 \mathrm{E}-02 \\
0.444 \mathrm{E}-02\end{array}$ & $\begin{array}{l}0.164 \mathrm{E}-02 \\
0.109 \mathrm{E}-02 \\
0.164 \mathrm{E}-02 \\
0.109 \mathrm{E}-02\end{array}$ & $\begin{array}{l}2.06 \\
2.04 \\
2.04 \\
2.03\end{array}$ & $\begin{array}{l}2.03 \\
2.02 \\
2.02 \\
2.02\end{array}$ \\
\hline Jensen & $\begin{array}{c}\text { Lax-Wendroff } \\
\text { Quickest }\end{array}$ & $\begin{array}{l}O\left(h^{2}\right) \\
O\left(h^{4}\right) \\
O\left(h^{2}\right) \\
O\left(h^{4}\right)\end{array}$ & $\begin{array}{c}0.925 \mathrm{E}-02 \\
0.154 \mathrm{E}-03 \\
0.915 \mathrm{E}-02 \\
0.153 \mathrm{E}-03\end{array}$ & $\begin{array}{l}0.223 \mathrm{E}-02 \\
0.930 \mathrm{E}-05 \\
0.222 \mathrm{E}-02 \\
0.930 \mathrm{E}-05\end{array}$ & $\begin{array}{l}0.546 \mathrm{E}-03 \\
0.571 \mathrm{E}-06 \\
0.546 \mathrm{E}-03 \\
0.571 \mathrm{E}-06\end{array}$ & $\begin{array}{l}2.06 \\
4.05 \\
2.04 \\
4.04\end{array}$ & $\begin{array}{l}2.03 \\
4.03 \\
2.03 \\
4.03\end{array}$ \\
\hline d'Allesio & $\begin{array}{c}\text { Lax-Wendroff } \\
\text { Quickest }\end{array}$ & $\begin{array}{l}O\left(h^{2}\right) \\
O\left(h^{4}\right) \\
O\left(h^{2}\right) \\
O\left(h^{4}\right)\end{array}$ & $\begin{array}{l}0.686 \mathrm{E}-01 \\
0.611 \mathrm{E}-01 \\
0.653 \mathrm{E}-01 \\
0.582 \mathrm{E}-01\end{array}$ & $\begin{array}{l}0.137 \mathrm{E}-01 \\
0.123 \mathrm{E}-01 \\
0.136 \mathrm{E}-01 \\
0.122 \mathrm{E}-01\end{array}$ & $\begin{array}{l}0.319 \mathrm{E}-02 \\
0.284 \mathrm{E}-02 \\
0.318 \mathrm{E}-02 \\
0.283 \mathrm{E}-02\end{array}$ & $\begin{array}{l}2.32 \\
2.31 \\
2.26 \\
2.26\end{array}$ & $\begin{array}{l}2.11 \\
2.11 \\
2.10 \\
2.10\end{array}$ \\
\hline Briley & $\begin{array}{c}\text { Lax-Wendroff } \\
\text { Quickest }\end{array}$ & $\begin{array}{l}O\left(h^{2}\right) \\
O\left(h^{4}\right) \\
O\left(h^{2}\right) \\
O\left(h^{4}\right)\end{array}$ & $\begin{array}{l}0.961 \mathrm{E}-02 \\
0.103 \mathrm{E}-03 \\
0.947 \mathrm{E}-02 \\
0.102 \mathrm{E}-03\end{array}$ & $\begin{array}{l}0.224 \mathrm{E}-02 \\
0.628 \mathrm{E}-05 \\
0.224 \mathrm{E}-02 \\
0.627 \mathrm{E}-05\end{array}$ & $\begin{array}{l}0.547 \mathrm{E}-03 \\
0.387 \mathrm{E}-06 \\
0.547 \mathrm{E}-03 \\
0.387 \mathrm{E}-06\end{array}$ & $\begin{array}{l}2.10 \\
4.04 \\
2.08 \\
4.02\end{array}$ & $\begin{array}{l}2.04 \\
4.02 \\
2.03 \\
4.02\end{array}$ \\
\hline
\end{tabular}

Numerical parameters are: $R e=100, \Delta t=0.0005$, multigrid residual less than $10^{-7}$. 


\section{DRIVEN CAVITY LAMINAR FLOW}

In this section, we consider time marching solutions for a full Navier-Stokes problem using the range of discretizations just described and present stability regions and some numerical results for accuracy. This problem has long served as the prototype for the incompressible Navier-Stokes equations; see, References [7,25-27], to mention only a few.

We assume $u=0$ and $v=0$ on all the fixed walls and that on the moving wall at $y=1$, $u=1$ and $v=0$. These boundary conditions can be written in terms of the stream function as

$$
\psi=0
$$

on all boundaries and

$$
\frac{\partial \psi}{\partial n}=\varepsilon
$$

where $\varepsilon=0$ on fixed walls and $\varepsilon=1$ on the moving wall at $y=1$. The coordinate $n$ is normal to the surface.

\subsection{Stability}

In the driven cavity problem, energy is provided to the system through forces acting on the moving wall and this energy is dissipated by viscous action, becoming heat which will be lost through the cavity walls. The driven cavity does not show stability for a Reynolds number larger than 7500, instead there is bounded oscillations of the energy even for very small time-steps. We have not analysed the nature of this oscillation, although the reasons may be associated with the dynamical features of the physical problem as reported in the literature for the driven cavity in References [27-29], where other numerical schemes were used.
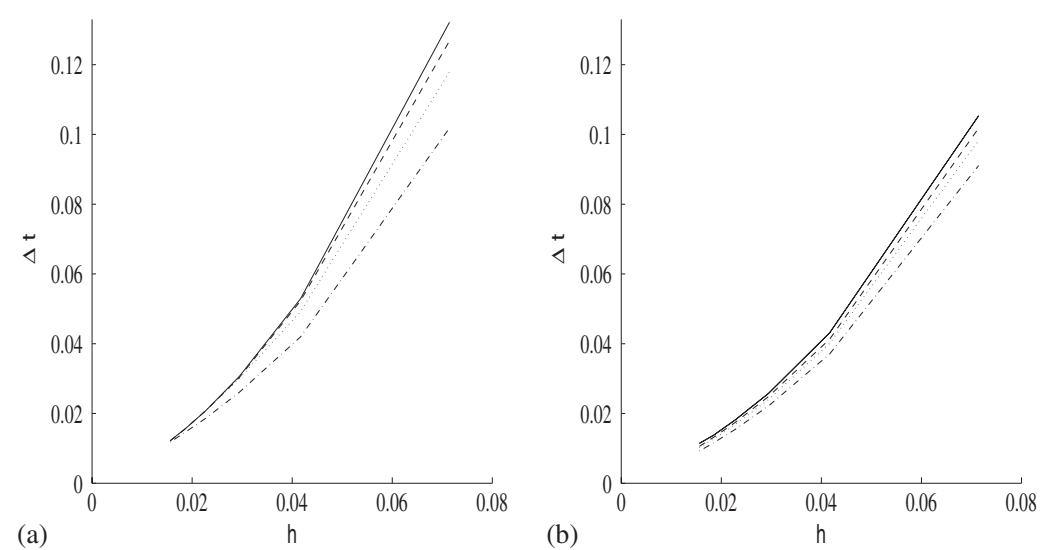

Figure 5. Unstable region for $R e=350$ [above the curves]: eigenvalues larger than one: (a) for the second-order method; (b) for the third-order method. Thom's boundary condition (-); Alessio's boundary condition $(-\cdot-)$; Wood's and Briley's boundary condition $(\cdots)$; Jensen's boundary condition $(--)$. 
Table III. Calculation of wall vorticity midway along moving wall, $\omega(0.5,1)$, for four mesh resolutions together with extrapolated convergence rate for varying vorticity boundary condition, convection-diffusion and stream function-vorticity equation discretization.

\begin{tabular}{|c|c|c|c|c|c|c|c|c|c|}
\hline \multirow[b]{2}{*}{$\left.\omega\right|_{\partial \Omega}$} & \multirow[b]{2}{*}{$\mathbf{u} \cdot \nabla \omega$} & \multirow[b]{2}{*}{$\nabla^{2} \psi=-\omega$} & \multicolumn{4}{|c|}{$\omega(0.5,1)$ reference $=6.564094$} & \multicolumn{3}{|c|}{ Convergence } \\
\hline & & & $32 \times 32$ & $64 \times 64$ & $128 \times 128$ & $256 \times 256$ & $32-64$ & $64-128$ & $128-256$ \\
\hline Thom & $\begin{array}{c}\text { Lax-Wendroff } \\
\text { Quickest }\end{array}$ & $\begin{array}{l}O\left(h^{2}\right) \\
O\left(h^{4}\right) \\
O\left(h^{2}\right) \\
O\left(h^{4}\right)\end{array}$ & $\begin{array}{l}6.990319 \\
6.973841 \\
6.856680 \\
6.838890\end{array}$ & $\begin{array}{l}6.659525 \\
6.650393 \\
6.630311 \\
6.620976\end{array}$ & $\begin{array}{l}6.585685 \\
6.582504 \\
6.581038 \\
6.577831\end{array}$ & $\begin{array}{l}6.569531 \\
6.568800 \\
6.568237 \\
6.567307\end{array}$ & $\begin{array}{l}2.16 \\
2.25 \\
2.14 \\
2.27\end{array}$ & $\begin{array}{l}2.14 \\
2.23 \\
1.97 \\
2.05\end{array}$ & $\begin{array}{l}1.99 \\
1.97 \\
2.03 \\
2.10\end{array}$ \\
\hline Woods & $\begin{array}{c}\text { Lax-Wendroff } \\
\text { Quickest }\end{array}$ & $\begin{array}{l}O\left(h^{2}\right) \\
O\left(h^{4}\right) \\
O\left(h^{2}\right) \\
O\left(h^{4}\right)\end{array}$ & $\begin{array}{l}6.856138 \\
6.833118 \\
6.727854 \\
6.703616\end{array}$ & $\begin{array}{l}6.636151 \\
6.626328 \\
6.607491 \\
6.597450\end{array}$ & $\begin{array}{l}6.581579 \\
6.578337 \\
6.576985 \\
6.573714\end{array}$ & $\begin{array}{l}6.568751 \\
6.568015 \\
6.567460 \\
6.566524\end{array}$ & $\begin{array}{l}2.02 \\
2.11 \\
1.92 \\
2.06\end{array}$ & $\begin{array}{l}2.04 \\
2.13 \\
1.75 \\
1.79\end{array}$ & $\begin{array}{l}1.91 \\
1.86 \\
1.94 \\
1.98\end{array}$ \\
\hline Jensen & $\begin{array}{c}\text { Lax-Wendroff } \\
\text { Quickest }\end{array}$ & $\begin{array}{l}O\left(h^{2}\right) \\
O\left(h^{4}\right) \\
O\left(h^{2}\right) \\
O\left(h^{4}\right)\end{array}$ & $\begin{array}{l}6.851832 \\
6.831085 \\
6.724270 \\
6.701177\end{array}$ & $\begin{array}{l}6.635290 \\
6.624681 \\
6.606717 \\
6.595836\end{array}$ & $\begin{array}{l}6.581445 \\
6.577995 \\
6.576860 \\
6.573376\end{array}$ & $\begin{array}{l}6.568735 \\
6.567962 \\
6.567444 \\
6.566472\end{array}$ & $\begin{array}{l}2.01 \\
2.14 \\
1.91 \\
2.11\end{array}$ & $\begin{array}{l}2.04 \\
2.12 \\
1.74 \\
1.77\end{array}$ & $\begin{array}{l}1.90 \\
1.85 \\
1.93 \\
1.96\end{array}$ \\
\hline d'Allesio & $\begin{array}{c}\text { Lax-Wendroff } \\
\text { Quickest }\end{array}$ & $\begin{array}{l}O\left(h^{2}\right) \\
O\left(h^{4}\right) \\
O\left(h^{2}\right) \\
O\left(h^{4}\right)\end{array}$ & $\begin{array}{l}6.915013 \\
6.889537 \\
6.780886 \\
6.754719\end{array}$ & $\begin{array}{l}6.665129 \\
6.655285 \\
6.635431 \\
6.625346\end{array}$ & $\begin{array}{l}6.592959 \\
6.589724 \\
6.588223 \\
6.584954\end{array}$ & $\begin{array}{l}6.573593 \\
6.572859 \\
6.572284 \\
6.571350\end{array}$ & $\begin{array}{l}1.80 \\
1.84 \\
1.60 \\
1.64\end{array}$ & $\begin{array}{l}1.81 \\
1.83 \\
1.56 \\
1.55\end{array}$ & $\begin{array}{l}1.60 \\
1.55 \\
1.56 \\
1.52\end{array}$ \\
\hline Briley & $\begin{array}{c}\text { Lax-Wendroff } \\
\text { Quickest }\end{array}$ & $\begin{array}{l}O\left(h^{2}\right) \\
O\left(h^{4}\right) \\
O\left(h^{2}\right) \\
O\left(h^{4}\right)\end{array}$ & $\begin{array}{l}6.846822 \\
6.823843 \\
6.721911 \\
6.696143\end{array}$ & $\begin{array}{l}6.638982 \\
6.627732 \\
6.610509 \\
6.598940\end{array}$ & $\begin{array}{l}6.582381 \\
6.578811 \\
6.577795 \\
6.574191\end{array}$ & $\begin{array}{l}6.568893 \\
6.567906 \\
6.567601 \\
6.566612\end{array}$ & $\begin{array}{l}1.92 \\
2.03 \\
1.77 \\
1.92\end{array}$ & $\begin{array}{l}2.03 \\
2.11 \\
1.76 \\
1.79\end{array}$ & $\begin{array}{l}1.93 \\
1.95 \\
1.97 \\
2.00\end{array}$ \\
\hline
\end{tabular}

The reference value given by Botella and Peyret [30] is $\omega(0.5,1)=6.564094$. Numerical parameters are: $R e=100$, $\Delta t=0.0005$, multigrid residual less than $10^{-7}$.

For the driven cavity problem, a steady laminar flow exists for $R e<3000$. In this section, we give calculations of the stability of numerical calculation of the steady state for a representative Reynolds number 350 .

We consider the matrix formulation, described in Section 2, of the system composed by the vorticity equation with the Poisson's equation. The velocity field $(\tilde{u}, \tilde{v})$ introduced in the vorticity equation is the numerical approximation velocity field to $(u, v)$ that we obtain from numerical solution of the cavity flow problem.

When we use the second- and third-order discretization for the vorticity equation and for Thom, Alessio and Dennis and Briley vorticity boundary discretizations at Reynolds number 350 , the result of the eigenvalues for the matrix formulation is described in Figure 5. We have used the second-order Poisson's discretization. Nevertheless if instead we use the fourth-order Poisson's discretization the stability results are very similar to the ones presented in Figure 5. 
Table IV. Calculation of vorticity at the centre, $\omega(0.5,0.5)$, for four mesh resolutions together with extrapolated convergence rate for varying vorticity boundary condition, convection-diffusion and stream function-vorticity equation discretization.

\begin{tabular}{|c|c|c|c|c|c|c|c|c|c|}
\hline \multirow{2}{*}{$\begin{array}{l}\text { Boundary } \\
\text { method }\end{array}$} & \multirow[b]{2}{*}{ Convection-diffusion } & \multirow{2}{*}{$\begin{array}{l}\text { Poisson } \\
\text { equation }\end{array}$} & \multicolumn{4}{|c|}{$\omega(0.5,0.5)$ reference $=1.174412$} & \multicolumn{3}{|c|}{ Convergence } \\
\hline & & & $32 \times 32$ & $64 \times 64$ & $128 \times 128$ & $256 \times 256$ & $32-64$ & $64-128$ & $128-256$ \\
\hline Thom & $\begin{array}{c}\text { Lax-Wendroff } \\
\text { Quickest }\end{array}$ & $\begin{array}{l}O\left(h^{2}\right) \\
O\left(h^{4}\right) \\
O\left(h^{2}\right) \\
O\left(h^{4}\right)\end{array}$ & $\begin{array}{l}1.063036 \\
1.087573 \\
1.106146 \\
1.131313\end{array}$ & $\begin{array}{l}1.146349 \\
1.153773 \\
1.157270 \\
1.164780\end{array}$ & $\begin{array}{l}1.166998 \\
1.169074 \\
1.168431 \\
1.170514\end{array}$ & $\begin{array}{l}1.172601 \\
1.173216 \\
1.173058 \\
1.173611\end{array}$ & $\begin{array}{l}1.99 \\
2.07 \\
1.99 \\
2.16\end{array}$ & $\begin{array}{l}1.92 \\
1.95 \\
1.52 \\
1.31\end{array}$ & $\begin{array}{l}2.03 \\
2.16 \\
2.14 \\
2.28\end{array}$ \\
\hline Woods & $\begin{array}{c}\text { Lax-Wendroff } \\
\text { Quickest }\end{array}$ & $\begin{array}{l}O\left(h^{2}\right) \\
O\left(h^{4}\right) \\
O\left(h^{2}\right) \\
O\left(h^{4}\right)\end{array}$ & $\begin{array}{l}1.081257 \\
1.106249 \\
1.123390 \\
1.148942\end{array}$ & $\begin{array}{l}1.149183 \\
1.156676 \\
1.160001 \\
1.167596\end{array}$ & $\begin{array}{l}1.167304 \\
1.169390 \\
1.168729 \\
1.170825\end{array}$ & $\begin{array}{l}1.172618 \\
1.173229 \\
1.173070 \\
1.173624\end{array}$ & $\begin{array}{l}1.88 \\
1.94 \\
1.82 \\
1.90\end{array}$ & $\begin{array}{l}1.83 \\
1.82 \\
1.34 \\
0.93\end{array}$ & $\begin{array}{l}1.99 \\
2.09 \\
2.08 \\
2.19\end{array}$ \\
\hline Jensen & $\begin{array}{l}\text { Lax-Wendroff } \\
\text { Quickest }\end{array}$ & $\begin{array}{l}O\left(h^{2}\right) \\
O\left(h^{4}\right) \\
O\left(h^{2}\right) \\
O\left(h^{4}\right)\end{array}$ & $\begin{array}{l}1.081077 \\
1.105883 \\
1.122976 \\
1.148631\end{array}$ & $\begin{array}{l}1.149267 \\
1.156949 \\
1.160052 \\
1.167852\end{array}$ & $\begin{array}{l}1.167335 \\
1.169465 \\
1.168758 \\
1.170904\end{array}$ & $\begin{array}{l}1.172620 \\
1.173245 \\
1.173078 \\
1.173040\end{array}$ & $\begin{array}{l}1.89 \\
1.97 \\
1.84 \\
1.97\end{array}$ & $\begin{array}{l}1.83 \\
1.82 \\
1.34 \\
0.90\end{array}$ & $\begin{array}{l}1.98 \\
2.08 \\
2.08 \\
1.35\end{array}$ \\
\hline d'Allesio & $\begin{array}{c}\text { Lax-Wendroff } \\
\text { Quickest }\end{array}$ & $\begin{array}{l}O\left(h^{2}\right) \\
O\left(h^{4}\right) \\
O\left(h^{2}\right) \\
O\left(h^{4}\right)\end{array}$ & $\begin{array}{l}1.063547 \\
1.088474 \\
1.106677 \\
1.132078\end{array}$ & $\begin{array}{l}1.140899 \\
1.148362 \\
1.151932 \\
1.159515\end{array}$ & $\begin{array}{l}1.163966 \\
1.166048 \\
1.165426 \\
1.167520\end{array}$ & $\begin{array}{l}1.171173 \\
1.171759 \\
1.171635 \\
1.172189\end{array}$ & $\begin{array}{l}1.73 \\
1.72 \\
1.59 \\
1.51\end{array}$ & $\begin{array}{l}1.68 \\
1.64 \\
1.32 \\
1.11 \\
\end{array}$ & $\begin{array}{l}1.69 \\
1.66 \\
1.69 \\
1.63\end{array}$ \\
\hline Briley & $\begin{array}{c}\text { Lax-Wendroff } \\
\text { Quickest }\end{array}$ & $\begin{array}{l}O\left(h^{2}\right) \\
O\left(h^{4}\right) \\
O\left(h^{2}\right) \\
O\left(h^{4}\right)\end{array}$ & $\begin{array}{l}1.081195 \\
1.106169 \\
1.122568 \\
1.148513\end{array}$ & $\begin{array}{l}1.148418 \\
1.156240 \\
1.159183 \\
1.167140\end{array}$ & $\begin{array}{l}1.167115 \\
1.169278 \\
1.168539 \\
1.170713\end{array}$ & $\begin{array}{l}1.172590 \\
1.173146 \\
1.173038 \\
1.173605\end{array}$ & $\begin{array}{l}1.84 \\
1.91 \\
1.77 \\
1.83\end{array}$ & $\begin{array}{l}1.83 \\
1.82 \\
1.37 \\
0.98\end{array}$ & $\begin{array}{l}2.00 \\
2.02 \\
2.10 \\
2.20\end{array}$ \\
\hline
\end{tabular}

The reference value given by Botella and Peyret [30] is $\omega(0.5,0.5)=1.174412$. Numerical parameters are: $R e=100$, $\Delta t=0.0005$, multigrid residual less than $10^{-7}$.

\subsection{Accuracy}

There are well established calculations of the driven cavity problem which provide reference values for the solution at some points in the flow. We use those from Reference [30] which were computed using a high-order spectral method. In particular, we examine the case $R e=100$ and the value of the vorticity in the middle of the moving wall and the vorticity and stream function in the centre of the cavity. The results were considered converged for a variation of the vorticity between time steps of order $10^{-21}$, that is the limit of double precision accuracy. We have used four meshes, $32 \times 32,64 \times 64,128 \times 128$ and $256 \times 256$. Then results from pairs of meshes were extrapolated to give an estimate of the convergence rate as the mesh size decreases. The results are given in Tables III-V. For most of the calculations the convergence rate is essentially quadratic. The exception is the condition from d'Allesion and Dennis where the convergence rate seems closer to 1.5. It is apparent that the choice of discretization of the Poisson equation makes no difference, the conditions which gave quar- 
Table V. Calculation of streamfunction at centre, $\psi(0.5,0.5)$, for four mesh resolutions together with extrapolated convergence rate for varying vorticity boundary condition, convection-diffusion and stream function-vorticity equation discretization.

\begin{tabular}{|c|c|c|c|c|c|c|c|c|c|}
\hline \multirow{2}{*}{$\begin{array}{l}\text { Boundary } \\
\text { method }\end{array}$} & \multirow[b]{2}{*}{ Convection-diffusion } & \multirow{2}{*}{$\begin{array}{l}\text { Poisson } \\
\text { equation }\end{array}$} & \multicolumn{4}{|c|}{$\psi(0.5,0.5)$ reference $=0.0665474$} & \multicolumn{3}{|c|}{ Convergence } \\
\hline & & & $32 \times 32$ & $64 \times 64$ & $128 \times 128$ & $256 \times 256$ & $32-64$ & $64-128$ & $128-256$ \\
\hline \multirow{4}{*}{ Thom } & \multirow{4}{*}{$\begin{array}{c}\text { Lax-Wendroff } \\
\text { Quickest }\end{array}$} & $O\left(h^{2}\right)$ & 0.062828 & 0.065634 & 0.066314 & 0.066490 & 2.03 & 1.97 & 2.02 \\
\hline & & $O\left(h^{4}\right)$ & 0.063660 & 0.065884 & 0.066385 & 0.066510 & 2.12 & 2.03 & 2.13 \\
\hline & & $O\left(h^{2}\right)$ & 0.064146 & 0.065960 & 0.066355 & 0.066503 & 2.03 & 1.61 & 2.13 \\
\hline & & $O\left(h^{4}\right)$ & 0.064976 & 0.066215 & 0.066426 & 0.066522 & 2.24 & 1.45 & 2.27 \\
\hline \multirow{4}{*}{ Woods } & \multirow{4}{*}{$\begin{array}{c}\text { Lax-Wendroff } \\
\text { Quickest }\end{array}$} & $O\left(h^{2}\right)$ & 0.063479 & 0.065729 & 0.066322 & 0.066490 & 1.91 & 1.86 & 1.96 \\
\hline & & $O\left(h^{4}\right)$ & 0.064325 & 0.065982 & 0.066393 & 0.066510 & 1.97 & 1.88 & 2.04 \\
\hline & & $O\left(h^{2}\right)$ & 0.064746 & 0.066051 & 0.066363 & 0.066503 & 1.86 & 1.43 & 2.05 \\
\hline & & $O\left(h^{4}\right)$ & 0.065596 & 0.066306 & 0.066434 & 0.066522 & 1.98 & 1.09 & 2.15 \\
\hline \multirow{4}{*}{ Jensen } & \multirow{4}{*}{$\begin{array}{c}\text { Lax-Wendroff } \\
\text { Quickest }\end{array}$} & $O\left(h^{2}\right)$ & 0.063 & 0.0 & 0.0 & 0.0 & 1.91 & 1.86 & 1.96 \\
\hline & & $O\left(h^{4}\right)$ & 0.064322 & 0.065993 & 0.066396 & 0.066510 & 2.01 & 1.88 & 2.03 \\
\hline & & $O\left(h^{2}\right)$ & 0.064742 & 0.066054 & 0.066364 & 0.066503 & 1.87 & 1.43 & 2.05 \\
\hline & & $O\left(h^{4}\right)$ & 0.065595 & 0.066317 & 0.066437 & 0.066523 & 2.05 & 1.06 & 2.15 \\
\hline \multirow{4}{*}{ d'Allesio } & \multirow{4}{*}{$\begin{array}{c}\text { Lax-Wendroff } \\
\text { Quickest }\end{array}$} & $O\left(h^{2}\right)$ & 0.0 & 0.0 & 0.0 & 0.0 & 1.72 & 1.68 & 1.63 \\
\hline & & $O\left(h^{4}\right)$ & 0.063566 & 0.065636 & 0.066254 & 0.066450 & 1.71 & 1.63 & 1.58 \\
\hline & & $O\left(h^{2}\right)$ & 0.064039 & 0.065717 & 0.066226 & 0.066443 & 1.59 & 1.37 & 1.62 \\
\hline & & $O\left(h^{4}\right)$ & 0.064885 & 0.065972 & 0.066297 & 0.066462 & 1.53 & 1.20 & 1.55 \\
\hline \multirow{4}{*}{ Briley } & \multirow{4}{*}{$\begin{array}{c}\text { Lax-Wendroff } \\
\text { Quickest }\end{array}$} & $O\left(h^{2}\right)$ & 0.063483 & 0. & 0.0 & 0.0 & 1.86 & & 1.98 \\
\hline & & $O\left(h^{4}\right)$ & 0.064328 & 0.065966 & 0.066389 & 0.066508 & 1.93 & 1.88 & 2.00 \\
\hline & & $O\left(h^{2}\right)$ & 0.064722 & 0.066021 & 0.066356 & 0.066502 & 1.79 & 1.46 & 2.07 \\
\hline & & $O\left(h^{4}\right)$ & 0.065587 & 0.066290 & 0.066430 & 0.066521 & 1.90 & 1.14 & 2.16 \\
\hline
\end{tabular}

Numerical parameters are: $R e=100, \Delta t=0.0005$, multigrid residual less than $10^{-7}$.

tic convergence in the previous section are now only quadratic so that the conditions for Bramble-Hubbard theory to allow global convergence to be determined by discretization in the interior do not hold (recall that Quickest should be close to third-order accurate in space so we might have hoped to find Quickest plus quartic discretization of the Poisson equation giving close to third-order convergence). As no global exact solution exists, convergence is considered only at three point values.

\section{CONCLUSION}

We have developed a global iteration matrix formulation for the stream-function vorticity equations and applied it to two driven cavity problems to examine the effect on numerical stability of different numerical vorticity boundary conditions. The main conclusion is that 
the various ways of treating the boundary vorticity make little difference to stability which is determined mainly by the discretization of the advection-diffusion vorticity equation. We have observed in passing some results for accuracy of the calculations and observe that there are situations where discretization errors near the boundary do not propagate into the interior and do not affect the global accuracy, as given by Bramble-Hubbard theory but for the most part, in solution of practical problems using time marching schemes, it is difficult to exceed second-order convergence.

\section{ACKNOWLEDGEMENTS}

ES was partially supported by Centro de Matemática da Universidade de Coimbra.

\section{REFERENCES}

1. Ottino J. The Kinematics of Mixing, Stretching, Chaos and Transport. Cambridge University Press: Cambridge, 1989.

2. Thom A. The flow past a circular cylinders at low speeds. Proceedings of the Royal Society of London 1933; A141:651-666.

3. Roache P. Computational Fluid Dynamics. Hermosa, 1972.

4. Peyret R, Taylor T. Computational Methods for Fluid Flow. Springer: Berlin, 1986.

5. Orszag S, Israeli M. Numerical simulation of viscous incompressible flows. Annual Review of Fluid Mechanics 1974; 6:281-318.

6. Gresho P. Some interesting issues in incompressible fluid dynamics, both in the continuum and in numerical simulation. Advances in Applied Mechanics 1992; 28:45-140.

7. Hou T, Wetton B. Convergence of a finite difference scheme for the Navier-Stokes equations using vorticity boundary conditions. SIAM Journal on Numerical Analysis 1992; 29:615-639.

8. Weinan E, Liu J. Essentially compact schemes for unsteady viscous incompressible flows. Journal of Computational Physics 1996; 126:122-138.

9. Wang C, Liu J. Analysis of finite difference schemes for unsteady Navier-Stokes equations in vorticity formulation. Numerische Mathematik 2002; 91:543-576.

10. Weinan E, Liu J. Vorticity boundary condition and related issues for finite difference schemes. Journal of Computational Physics 1996; 124:368-382.

11. Napolitano M, Pascazio G, Quartapelle L. A review of vorticity conditions in the numerical solution of the $\omega-\psi$ equations. Computer and Fluids 1999; 28:139-185.

12. Li Z, Wang C. A fast finite difference method for solving Navier-Stokes equations on irregular domains. Communications in Mathematical Sciences 2003; 1:180-196.

13. Spotz W. Accuracy and performance of numerical wall boundary conditions for steady, 2D incompressible stream function vorticity. International Journal for Numerical Methods in Fluids 1998; 28:737-757.

14. Sousa E, Sobey I. Numerical stability of unsteady stream-function vorticity calculations. Communications in Numerical Methods in Engineering 2003; 19:407-419.

15. Richtmyer R, Morton K. Difference Methods for Initial-value Problems. Wiley: New York, 1967.

16. Sousa E, Sobey I. A family of finite difference schemes for the convection-diffusion equation in two dimensions. Enumath 2001 Numerical Mathematics and Advanced Applications. Springer: Berlin, 2002; 179-188.

17. Iserles A. A First Course in the Numerical Analysis of Differential Equations. Cambridge University Press: Cambridge, 1996.

18. Woods L. A note on the numerical solution of a fourth order differential equation. Aeronautical Quarterly 1954; 5:176-182.

19. Jensen V. Viscous flow round a sphere at low Reynolds numbers. Proceedings of the Royal Society 1959; A294:346-366.

20. d'Alessio S, Dennis S. A vorticity model for viscous flow past a cylinder. Computers and Fluids 1994; 23:279-293.

21. Briley W. A numerical study of laminar separation bubbles using Navier-Stokes equations. Journal of Fluid Mechanics 1971; 47:713-736.

22. Turkel E. Symmetric hyperbolic difference schemes and matrix problems. Linear Algebra and its Applications 1977; 16:109-129.

23. Collatz L. Numerische behandlung von differentialgleichungen. Springer: Berlin, 1951. 
24. Bramble J, Hubbard B. New monotone type approximations for elliptic problems. Mathematics of Computation $1964 ; 18: 349-367$.

25. Benjamin A, Denny V. On the convergence of numerical solutions for 2-D flows in a cavity at large Re. Journal of Computational Physics 1979; 33:340-358.

26. Schreiber R, Keller H. Driven cavity flows by efficient numerical techniques. Journal of Computational Physics 1983; 49:310-333.

27. Shen J. Hopf bifurcation of the unsteady regularized driven cavity flow. Journal of Computational Physics 1991; 95:228-245.

28. Bruneau C, Jouron C. A new upwind scheme for the driven cavity flow. Comptes Rendus des Academie des Sciences 1988; 307:359-362.

29. Goodrich J, Gustafson K, Halasi K. Hopf bifurcation in the driven cavity. Journal of Computational Physics 1990; 90:219-261.

30. Botella O, Peyret R. Benchmark spectral results on the lid-driven cavity flow. Computers and Fluids 1998; 27:421-433. 